\title{
Optical properties of new zirconia-based dental ceramics: literature review
}

\author{
G. Pizzolatto ${ }^{1}$, M. Borba ${ }^{1 *}$ \\ ${ }^{1}$ University of Passo Fundo, Dental School, Post-Graduation Program in Dentistry, BR286, 99052-900, \\ Passo Fundo, RS, Brazil
}

\begin{abstract}
The first generation of polycrystalline zirconia-based ceramic used to produce dental prosthesis showed excellent mechanical behavior; however, its low translucency limited the indication for aesthetic monolithic restorations. Thus, various strategies have been tested in order to develop a material that has high strength and good optical properties. The aim of this study was to characterize, through a literature review, the translucency of different types of zirconia-based ceramics indicated for monolithic restorations. The literature search was performed in PubMed/Medline database, from 2010 to 2019, using as keywords: zirconia, translucency, and optical properties. The $3^{\text {rd }}$ (monochromatic) and $4^{\text {th }}$ (multichromatic) generations of zirconia, which have high cubic phase content, showed the highest translucency among the zirconia-based ceramics investigated. Additionally, other strategies, such as the addition of lanthanum oxide and the infiltration of feldspathic glass in the zirconia surface (graded-zirconia), resulted in a good balance of optical and mechanical properties.
\end{abstract}

Keywords: ceramics, dental prosthesis, optical properties.

\section{INTRODUCTION}

For a long time, the metal-ceramic fixed dental prosthesis was the gold standard in dentistry. However, the gray color of the metal infrastructure does not resemble the shade and translucency of the dental structures at all. Therefore, all-ceramic prostheses became popular for their favorable biocompatibility and aesthetics [1, 2]. These factors are extremely important for patients, who seek dental prostheses with optical behavior similar to the tooth. Despite having these advantages, ceramics are brittle and susceptible to fractures. The development of dental materials that are both aesthetic and resistant to fracture is the goal of manufacturers and researchers [13].

The excellent mechanical properties of $1^{\text {st }}$ generation zirconia made it an attractive material to produce dental prostheses [3-7]. Pure zirconia has three main phases: monoclinic, tetragonal, and cubic, which are stable up to $1170{ }^{\circ} \mathrm{C}$, between $1170{ }^{\circ} \mathrm{C}$ and $2370{ }^{\circ} \mathrm{C}$, and above $2370^{\circ} \mathrm{C}$, respectively. However, the monoclinic phase does not have good mechanical properties. In order to improve the ceramic's mechanical behavior, stabilizing oxides, such as yttrium oxide $\left(\mathrm{Y}_{2} \mathrm{O}_{3}\right)$, are incorporated in its composition so the tetragonal phase remains stable even at room temperature $[8,9]$. The tetragonal phase can result in a material with superior fracture strength and toughness due to a phase transformation toughening

*marciaborba@upf.br

Dhttps://orcid.org/0000-0001-7587-9839 mechanism, where a reversion of the tetragonal to the monoclinic phase $(\mathrm{T} \rightarrow \mathrm{M})$ occurs when the material undergoes external stresses $[3,8,9]$. The $3 \mathrm{~mol} \%$ yttriastabilized tetragonal zirconia polycrystal (3Y-TZP) has exceptional mechanical properties and has been considered a promising material to produce all-ceramic single crowns and fixed partial dentures $[4,6]$. The biggest limitation is its opacity [5], caused mainly by the fact that its grains are birefringent and light is scattered at the grain boundaries [3]. Therefore, $1^{\text {st }}$ generation zirconia was initially indicated for fixed dental prostheses combined with a more aesthetic feldspathic porcelain or glass-ceramic veneer. Nevertheless, high chipping rates were reported for these multi-layer zirconia prostheses, especially involving the weak veneer $[10,11]$.

The monolithic all-ceramic prosthesis could result in lower chipping rates as the veneer layer is not present [3, 7]. Thus, in order to improve the aesthetics of zirconia polycrystalline ceramic, the concentration of the alumina sintering additive was reduced, and the porosity was eliminated by sintering the ceramic at higher temperatures [12]. These modifications originated the $2^{\text {nd }}$ generation of 3 Y-TZP ceramic and allowed to produce monolithic posterior prosthesis $[3,7]$. Still seeking greater aesthetics, the $3^{\text {rd }}$ generation of zirconia-based ceramics was developed. For this generation, the cubic phase content of the material was increased by adding a greater amount of stabilizing oxides to produce partially stabilized zirconias with 4 and $5 \mathrm{~mol} \%$ yttria. Although the translucency of the material was improved, the strength and fracture toughness were compromised, as cubic grains are not capable of undergoing phase transformation under stress $[13,14]$. Therefore, $3^{\text {rd }}$ generation zirconias are indicated 
to produce veneers, inlays, onlays, crowns, and anterior prostheses. Currently, a $4^{\text {th }}$ generation multichromatic zirconia with shade and translucency gradients is also available to produce monolithic restorations. These systems can have a single composition or a gradient of compositions $[15,16]$. Other strategies were also proposed to improve the optical properties of zirconiabased ceramics $[13,17]$ and are discussed further.

Restorative materials depend on different optical properties to mimic the natural tooth. These properties go beyond the shade of the restoration, which consists of its value, hue, and chroma. An essential optical property for the restoration to simulate the appearance of dental structures is translucency [18, 19]. The translucency of a ceramic is usually expressed in the studies by the translucency parameter (TP) or the contrast ratio (CR). $\mathrm{TP}$ is measured by evaluating the color difference between a material of uniform thickness on a black and a white background. A higher TP value corresponds to more translucent material. CR is measured by the relation between the spectral reflectance of light in a sample on black and white backgrounds, in which the most translucent material has a value of 0 and the most opaque has a value of 1 . The translucency of a ceramic material is related to the amount of light that it scatters, so if most of the light that passes through the ceramic is scattered or reflected the material becomes opaque, if only part of this light is scattered or reflected it becomes translucent $[18,20]$.

Considering the large number of strategies used to develop zirconia-based ceramics for monolithic restorations, it is important to verify whether the optical behavior of these new materials has been improved in relation to the conventional 3Y-TZP ( $1^{\text {st }}$ generation). Therefore, the aim of the present study was to characterize, through a literature review, the translucency of different types of zirconia-based ceramics indicated to produce monolithic prostheses.

\section{METHODS}

A literature search was performed using PubMed/ Medline database from 2010 to 2019 including only dental journals, using the keywords: zirconia, translucency, and optical properties. The inclusion criteria were laboratory studies that evaluated the optical properties of at least one of the new generations of zirconia-based ceramics indicated to produce monolithic prosthesis $\left(2^{\text {nd }}, 3^{\text {rd }}\right.$, and $4^{\text {th }}$ generations). Exclusion criteria were studies that investigated variables other than the ceramic materials, such as the luting agent, background, surface polishing methods, and thermal treatments. Fifty-five records were retrieved in the initial search. After title and abstract screening, 40 records were excluded. Fifteen full-text articles were evaluated and eight were included in the literature review. Fig. 1 shows the literature review flow diagram. Two researchers were involved in the screening of articles and data extraction.

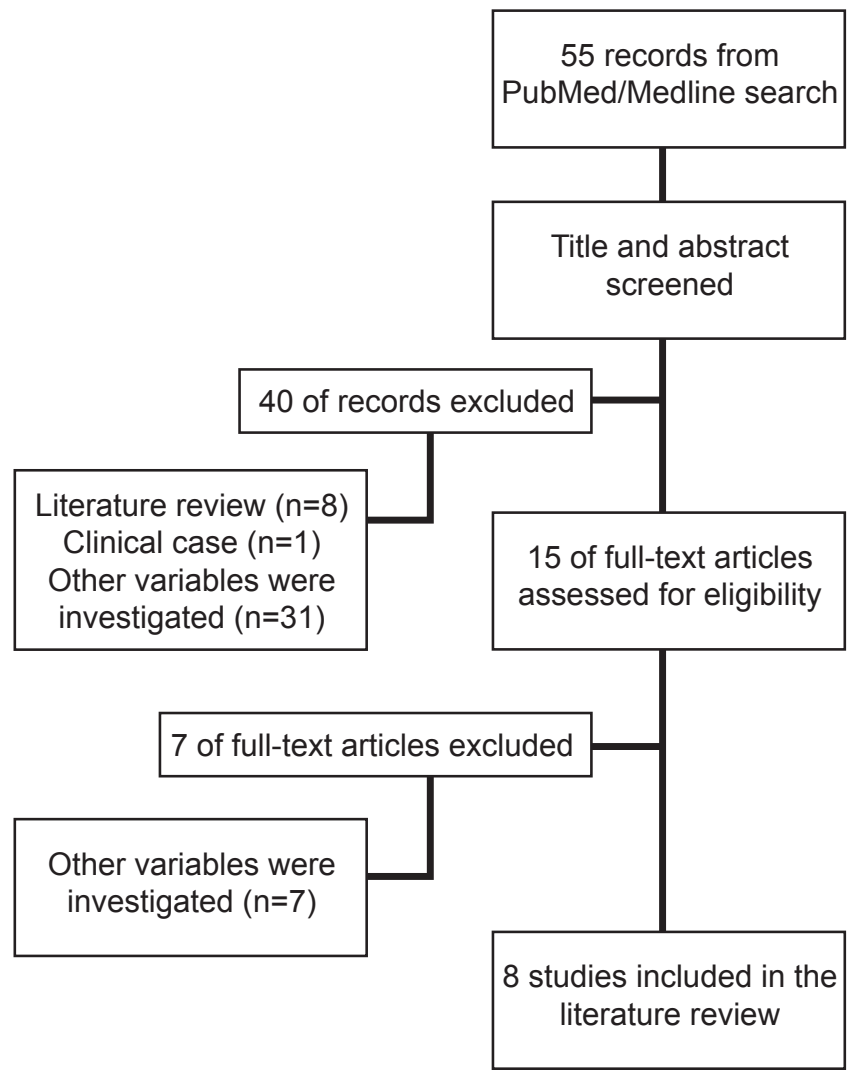

Figure 1: Literature review flow diagram.

\section{RESULTS}

Eight studies that met the inclusion and exclusion criteria were selected. Six studies evaluated the CR and the TP of different types of zirconia, one analyzed only the TP, and the other only the CR. Two of the eight studies evaluated zirconia-based ceramics of different thicknesses $[18,20]$, one also evaluated experimental zirconias [13], and another the graded zirconias [17]. One study investigated different veneering techniques [21]. The studies' main findings are shown in Table I.

\section{DISCUSSION}

Ceramic restorations in a monolithic configuration are desired for prosthetic rehabilitations. Zirconia polycrystals have proven to be a highly durable material, due to their excellent mechanical properties. However, they have not yet been able to achieve the optical properties of feldspathic porcelain and other glass-ceramics, which have superior translucency due to the greater amount of glass phase on their composition [19]. On the other hand, ceramics with high glassy content have lower fracture strength and toughness and are more susceptible to slow crack growth than polycrystalline ceramics $[1,2,6]$. Thus, in order to provide materials with good mechanical and optical properties, $2^{\text {nd }}, 3^{\text {rd }}$, and $4^{\text {th }}$ generations of zirconia emerged, and experimental zirconias with different compositions 
Table I - Data from in vitro studies of zirconia ceramics: translucency parameter (TP) and contrast ratio (CR).

\begin{tabular}{|c|c|c|c|c|c|}
\hline \multirow{2}{*}{ Material (brand) } & \multirow{2}{*}{ Generation/type } & \multirow{2}{*}{$\begin{array}{c}\text { Thick- } \\
\text { ness }\end{array}$} & \multicolumn{2}{|c|}{ Optical property } & \multirow{2}{*}{ Ref. } \\
\hline & & & $\mathrm{CR}^{*}$ & TP* & \\
\hline \multirow{2}{*}{ 5Y-PSZ (Prettau; Zirkonzahn) } & \multirow{2}{*}{$3^{\text {rd }}$} & $0.5 \mathrm{~mm}$ & 0.85 & 17.13 & \multirow{10}{*}[20]{} \\
\hline & & $1.0 \mathrm{~mm}$ & 0.90 & 12.46 & \\
\hline \multirow{2}{*}{ 5Y-PSZ (Bruxzir, Glidewell) } & \multirow{2}{*}{$3^{\text {rd }}$} & $0.5 \mathrm{~mm}$ & 0.86 & 17.76 & \\
\hline & & $1.0 \mathrm{~mm}$ & 0.92 & 12.32 & \\
\hline \multirow{2}{*}{ 4Y-PSZ (Wieland Zenostar, Ivoclar Vivadent) } & \multirow{2}{*}{$3^{\text {rd }}$} & $0.5 \mathrm{~mm}$ & 0.84 & 18.90 & \\
\hline & & $1.0 \mathrm{~mm}$ & 0.88 & 13.95 & \\
\hline \multirow{2}{*}{ 4Y-PSZ (Katana, Kuraray Noritake) } & \multirow{2}{*}{$3^{\text {rd }}$} & $0.5 \mathrm{~mm}$ & 0.84 & 18.23 & \\
\hline & & $1.0 \mathrm{~mm}$ & 0.87 & 14.51 & \\
\hline \multirow{2}{*}{ Y-FSZ (Prettau Anterior, Zirkonzahn) } & \multirow{2}{*}{$3^{\text {rd }}$} & $0.5 \mathrm{~mm}$ & 0.82 & 20.40 & \\
\hline & & $1.0 \mathrm{~mm}$ & 0.85 & 15.82 & \\
\hline
\end{tabular}

Conclusion: FSZ is relatively more translucent than PSZ; translucency of all the tested materials decreased as the thickness

\begin{tabular}{cccc} 
& increased & & \\
\hline 3Y-TZP (Ceramill ZI, Amann Girrbach) & $1^{\text {st }}$ & $0.5 \mathrm{~mm}$ & $0.77 \pm 0.01$ \\
3Y-TZP (Zenostar, Wieland) & $2^{\text {nd }}$ & $0.5 \mathrm{~mm}$ & $0.57 \pm 0.01$ \\
3Y-TZP (DD Bio ZX & $2^{\text {nd }}$ & $0.5 \mathrm{~mm}$ & $0.62 \pm 0.01$ \\
3Y-TZP (InCoris TZI, Sirona) & $2^{\text {nd }}$ & $0.5 \mathrm{~mm}$ & $0.57 \pm 0.01$ \\
3Y-TZP (Ceramill Zolid, Amann Girrbach) & $2^{\text {nd }}$ & $0.5 \mathrm{~mm}$ & $0.57 \pm 0.01$
\end{tabular}

Conclusion: $2^{\text {nd }} \mathrm{g}$. zirconia showed better optical properties, with a lower CR than the $1^{\text {st }} \mathrm{g}$.; no correlation was found between grain size and CR

$\begin{array}{ccccc}\text { 3Y-0.05Al (Zpex, Tosoh) } & 2^{\text {nd }} & 0.5 \mathrm{~mm} & 0.55 \pm 0.02 & 18.9 \pm 0.9 \\ 3 \mathrm{Y}-0.25 \mathrm{Al}(\mathrm{TZ}-3 \mathrm{Y}, \text { Tosoh) } & 1^{\text {st }} & 0.5 \mathrm{~mm} & 0.61 \pm 0.01 & 15.9 \pm 0.3 \\ \text { 3Y-0.10Al-0.2La } & \text { Experimental } & 0.5 \mathrm{~mm} & 0.48 \pm 0.01 & 22.6 \pm 0.6 \\ 3 \mathrm{Y}-0.25 \mathrm{Al}-0.2 \mathrm{La} & \text { Experimental } & 0.5 \mathrm{~mm} & 0.52 \pm 0.01 & 20.9 \pm 0.5 \\ 5 \mathrm{Y}-0.05 \mathrm{Al} \text { (Zpex Smile, Tosoh) } & 3^{\text {rd }} & 0.5 \mathrm{~mm} & 0.36 \pm 0.03 & 30.1 \pm 2.3\end{array}$

5Y-0.05Al (Zpex Smile, Tosoh)

Conclusion: decreasing the amount of alumina below $0.25 \mathrm{wt} \%$ prevents the formation of particles that disperse light and increases translucency; addition of $\mathrm{La}_{2} \mathrm{O}_{3}$ significantly improves translucency but is not as effective as adding cubic phase

\begin{tabular}{|c|c|c|c|c|}
\hline $\begin{array}{c}\text { Y-FSZ (Ceramill Zolid FX Multilayer, Amann } \\
\text { Girrbach) }\end{array}$ & $4^{\text {th }} ;$ multichromatic & $1.0 \mathrm{~mm}$ & $0.56 \pm 0.02$ & $19.4 \pm 0.5$ \\
\hline Y-FSZ (Prettau Anterior, Zirkonzahn) & $3^{\text {rd }}$ & $1.0 \mathrm{~mm}$ & $0.74 \pm 0.03$ & $16.8 \pm 0.4$ \\
\hline 4Y-PSZ (Zenostar T, Wieland) & $3^{\text {rd }}$ & $1.0 \mathrm{~mm}$ & $0.76 \pm 0.03$ & $15.9 \pm 0.4$ \\
\hline
\end{tabular}

Conclusion: multichromatic FSZ showed higher TP and lower CR; FSZ with a high amount of cubic phase shows greater translucency than PSZ

\begin{tabular}{|c|c|c|c|c|}
\hline 5Y-PSZ (Zpex Smile, Tosoh) & $3^{\text {rd }}$ & $1.0 \mathrm{~mm}$ & $0.34 \pm 0.02$ & $32.8 \pm 1.4$ \\
\hline 5Y-PSZ-P (Zpex Smile, Tosoh) & $3^{\text {rd }} ;$ polished & $1.0 \mathrm{~mm}$ & $0.31 \pm 0.01$ & $34.2 \pm 0.4$ \\
\hline 5Y-PSZ-AA (Zpex Smile, Tosoh) & $3^{\text {rd}} ;$ air-abraded & $1.0 \mathrm{~mm}$ & $0.38 \pm 0.01$ & $30.0 \pm 0.7$ \\
\hline 5Y-PSZ-G (Zpex Smile, Tosoh) & $3^{\text {rd }}$; glazed & $1.0 \mathrm{~mm}$ & $0.34 \pm 0.02$ & $33.8 \pm 1.4$ \\
\hline 5Y-PSZ-YGI (Zpex Smile, Tosoh) & $\begin{array}{c}3^{\text {rd; }} \text { glass-infiltrated } \\
\text { (yellow) }\end{array}$ & $1.0 \mathrm{~mm}$ & $0.32 \pm 0.00$ & $33.6 \pm 0.2$ \\
\hline 5Y-PSZ-MGI (Zpex Smile, Tosoh) & $\begin{array}{l}3^{\text {rd }} \text {; glass-infiltrated } \\
\text { (mixed) }\end{array}$ & $1.0 \mathrm{~mm}$ & $0.37 \pm 0.00$ & $29.2 \pm 0.1$ \\
\hline 5Y-PSZ-WGI (Zpex Smile, Tosoh) & $\begin{array}{l}3^{\text {rd }} \text {; glass-infiltrated } \\
\text { (white) }\end{array}$ & $1.0 \mathrm{~mm}$ & $0.38 \pm 0.00$ & $30.1 \pm 0.1$ \\
\hline 3Y-TZP (Zpex, Tosoh) & $2^{\text {nd }}$ & $1.0 \mathrm{~mm}$ & $0.48 \pm 0.00$ & $16.3 \pm 1.0$ \\
\hline
\end{tabular}

Conclusion: glass-infiltration of $3^{\text {rd }} \mathrm{g}$. zirconia allowed the creation of a material with a gradient of properties; this infiltration managed to maintain the optical properties of 5Y-PSZ and to increase the mechanical properties

$\begin{array}{ccccc}\text { 3Y-TZP (Aadva ST, GC Tech) } & 1^{\text {st }} & 1.0 \mathrm{~mm} & 0.74 \pm 0.01 & 36.9 \pm 0.1 \\ \text { 3Y-TZP 2 (Aadva EI, GC Tech) } & 2^{\text {nd }} & 1.0 \mathrm{~mm} & 0.70 \pm 0.01 & 38.4 \pm 0.1 \\ \text { 5Y-PSZ (Aadva NT, GC Tech) } & 3^{\text {rd }} & 1.0 \mathrm{~mm} & 0.62 \pm 0.01 & 43.4 \pm 0.1 \\ \text { SZ (Katana UTML, Kuraray Noritake) } & 4^{\text {th }} \text {; multichromatic } & 1.0 \mathrm{~mm} & 0.69 \pm 0.01 & 36.0 \pm 0.1\end{array}$

5Y-PSZ (Katana UTML, Kuraray Noritake)

Conclusion: a greater amount of cubic phase in the composition of the zirconia makes it more translucent; although there is a significant aesthetic improvement when comparing the groups, all four materials are considered to be of medium translucency 


\begin{tabular}{|c|c|c|c|c|c|}
\hline \multirow{2}{*}{ 3Y-TZP (VITA YZ-HT, Vita Zahnfabrik) } & \multirow{2}{*}{$2^{\text {nd }}$} & $0.5 \mathrm{~mm}$ & 0.53 & 19 & \multirow{8}{*}[18]{} \\
\hline & & $1.0 \mathrm{~mm}$ & 0.67 & 16 & \\
\hline \multirow{2}{*}{ 4Y-PSZ (VITA YZ-ST, Vita Zahnfabrik) } & \multirow{2}{*}{$3^{\text {rd }}$} & $0.5 \mathrm{~mm}$ & 0.43 & 24 & \\
\hline & & $1.0 \mathrm{~mm}$ & 0.41 & 22 & \\
\hline \multirow{2}{*}{ 5Y-PSZ (VITA YZ-XT, Vita Zahnfabrik) } & \multirow{2}{*}{$3^{\text {rd }}$} & $0.5 \mathrm{~mm}$ & 0.32 & 28 & \\
\hline & & $1.0 \mathrm{~mm}$ & 0.30 & 24 & \\
\hline \multirow{2}{*}{ LD (IPS e.max CAD LT, Ivoclar Vivadent) } & & $0.5 \mathrm{~mm}$ & 0.29 & 31 & \\
\hline & & $1.0 \mathrm{~mm}$ & 0.36 & 26 & \\
\hline \multicolumn{6}{|c|}{$\begin{array}{l}\text { Conclusion: differences in optical properties are related to variations in the amount of yttria, phase composition, and grain size; } \\
\text { translucency is exponentially related to the thickness of the material; 5Y-PSZ presented optical properties comparable to LD }\end{array}$} \\
\hline 3Y-TZP (IPS e.max Zircad, Ivoclar Vivadent) & $\begin{array}{l}2^{\text {nd. }} \text { glass-ceramic } \\
\text { (layering-LV) }\end{array}$ & $1.0 \mathrm{~mm}$ & & $9.5 \pm 0.9$ & \multirow{6}{*}[21]{} \\
\hline 3Y-TZP (IPS e.max Zircad, Ivoclar Vivadent) & $\begin{array}{l}2^{\text {nd, }} \text { glass-ceramic } \\
\text { (overpressing-OP) }\end{array}$ & $1.0 \mathrm{~mm}$ & & $11.2 \pm 1.2$ & \\
\hline 3Y-TZP (IPS e.max Zircad, Ivoclar Vivadent) & $\begin{array}{l}2^{\text {nd; }} \text { LD (porcelain } \\
\text { fused to zirconia) }\end{array}$ & $1.0 \mathrm{~mm}$ & & $11.8 \pm 0.8$ & \\
\hline 3Y-TZP (IPS e.max Zircad, Ivoclar Vivadent) & $\begin{array}{l}2^{\text {ndi }} ; \text { glass-ceramic } \\
\quad(\text { cutback-CB })\end{array}$ & $1.0 \mathrm{~mm}$ & & $10.3 \pm 0.8$ & \\
\hline 3Y-TZP (IPS e.max Zircad, Ivoclar Vivadent) & $2^{\text {nd }}$ (monolithic) & $1.0 \mathrm{~mm}$ & & $8.5 \pm 0.9$ & \\
\hline 4Y-PSZ (IPS e.max Zircad, Ivoclar Vivadent) & $3^{\text {rd }}$ (monolithic) & $1.0 \mathrm{~mm}$ & & $13.0 \pm 0.9$ & \\
\hline \multicolumn{6}{|c|}{$\begin{array}{l}\text { Conclusion: different manufacturing techniques affect the optical properties of zirconia restorations; } 4 \text { Y-PSZ was the most } \\
\text { translucent; among the veneering techniques, OP showed the highest TP and CB the lowest }\end{array}$} \\
\hline
\end{tabular}

*mean \pm standard deviation; FSZ: fully-stabilized zirconia; PSZ: partially-stabilized zirconia; LD: lithium disilicate glass-ceramic.

and addition of a glassy phase (graded-zirconia) were also developed.

Restorations of conventional $1^{\text {st }}$ generation 3Y-TZP are veneered with a more aesthetic ceramic. Different techniques are available to apply the feldspathic porcelain or glass-ceramic over the zirconia infrastructure, including conventional (layering-veneering) and partial stratification (cutback) techniques, hot injection (overpressure), and cementation or fusion of the two ceramic layers produced by CAD-CAM (fused porcelain to zirconia) [2]. Therefore, a study compared the translucency parameter (TP) of specimens produced using these different techniques with the TP of two zirconia-based ceramics for monolithic restorations, a low translucency 3Y-TZP, and a medium translucency 4Y-PSZ. They observed that $3^{\text {rd }}$ generation 4Y-PSZ had the highest TP, even when evaluated with the same thickness of the multi-layer specimens. Thus, if a more translucent restoration is required, $3^{\text {rd }}$ generation zirconia monolithic prosthesis can be used, which will promote a better aesthetic result than multi-layer zirconia veneered with porcelain or glass-ceramic. On the other hand, the conventional 3Y-TZP showed the lowest TP value, meaning that veneering with a more translucent ceramic is required to obtain an adequate aesthetic result [21].

When the $1^{\text {st }}$ and $2^{\text {nd }}$ generations of zirconia were compared, it was concluded that the $1^{\text {st }}$ generation is more opaque, but has better mechanical properties [12]. The study also noted that the correlation between translucency and grain size exists only in the $1^{\text {st }}$ generation, where the larger the grain, the greater the translucency. In the $2^{\text {nd }}$ generation, no correlation was found between the two parameters. In the $2^{\text {nd }}$ generation, the amount of alumina sintering additive is reduced and the zirconia grain size is comparable with the grain size of the $1^{\text {st }}$ generation [12]. $3^{\text {rd }}$ and $4^{\text {th }}$ generation cubic zirconias are available as partially stabilized (PSZ) or fully stabilized (FSZ) materials. Literature reports that FSZ has yttria content varying from $8.5 \%$ to $12 \%$, resulting in a higher amount of cubic phase than PSZ, which has $4 \%$ to $6 \%$ of yttria content. Studies showed that FSZ has higher TP than PSZ, mainly due to the differences in composition and microstructure [20, 22]. Nevertheless, these studies did not evaluate the cubic content either the composition of FSZ and PSZ materials.

Another investigation also analyzed the correlation between grain size and the optical properties of the different generations of zirconia [23]. Differences were found in the microstructure, composition, and optical properties of the studied materials. The $3^{\text {rd }}$ generation zirconia showed the greater TP value. When comparing $3^{\text {rd }}$ and $4^{\text {th }}$ generations, smaller grain size and higher TP were found for $3^{\text {rd }}$ generation monochromatic zirconia compared to $4^{\text {th }}$ generation multichromatic zirconia. Both materials were PSZ with high cubic content but different sintering protocols. In addition, the chroma gradient could also be responsible for decreasing translucency. Although there is a substantial improvement in translucency for $3^{\text {rd }}$ generation zirconia, all materials were considered to be of medium translucency [19].

Lithium disilicate glass-ceramic (LD) can be considered the gold standard for monolithic restorations as it provides a good balance between optical and mechanical properties [2]. Therefore, most studies compare the mechanical and optical behavior of zirconia-based ceramics with LD [24]. When evaluating 3Y-TZP, 4Y-PSZ, and 5Y-PSZ, an investigation observed a progressive increase in the translucency 
parameter with increasing grain size, yttria content, and cubic phase [18]. Furthermore, they concluded that LD has the highest translucency parameter, followed by 5Y-PSZ. All materials had a decrease in their TP and an increase in the contrast ratio (CR) as the thickness increased, corroborating a previous study [20]. Yet, the TP of $2^{\text {nd }}$ generation 3Y-TZP and LD showed a higher thickness dependence than $3^{\text {rd }}$ generation 4 Y-PSZ and 5Y-PSZ. For $2^{\text {nd }}$ generation 3Y-TZP and LD, the TP increased $26 \%$ and $24 \%$, respectively, when the thickness increased from 0.5 to $1 \mathrm{~mm}$, while for the $3^{\text {rd }}$ generation zirconias TP was mostly constant.

As mentioned, other strategies were also proposed aiming to improve the optical properties of zirconia-based ceramic without compromising their mechanical behavior [13, 17]. A study investigated different strategies, such as reducing the alumina content, increasing the yttria content, and adding lanthanum oxide $\left(\mathrm{La}_{2} \mathrm{O}_{3}\right)$. It was observed that reducing alumina to less than $0.25 \mathrm{wt} \%$ prevents the formation of particles that act as light scattering centers. However, completely eliminating alumina is not a good option as it is an important additive of the sintering process and has proven to be effective in preventing hydrothermal degradation. In addition, alumina-free zirconia had no difference in translucency, showing that its total densification is already sufficient to obtain a more translucent material (which occurs when alumina is reduced to $0.25 \%$ ). $3^{\text {rd }}$ generation zirconia, which had 54\% cubic and $46 \%$ tetragonal phases, was more translucent than the other materials and it is resistant to hydrothermal degradation; however, its mechanical properties have been drastically reduced. The addition of $0.2 \mathrm{~mol} \%$ of lanthanum oxide to $3 \mathrm{Y}$-TZP with $0.25 \mathrm{wt} \%$ alumina increased the translucency and resistance to degradation and maintained the phase transformation toughening mechanism, keeping the material's good mechanical properties. The mechanism that allows for greater translucency when adding lanthanum oxide is not yet fully understood, but it is known that it chemically changes the grain boundaries by segregation [13].

Another tested strategy was to infiltrate a feldspathic glass of different shades in the surface of $3^{\text {rd }}$ generation 5Y-PSZ, creating a material with a gradient of elastic properties, called graded-zirconia. The surface glass infiltration increased the flexural strength of 5Y-PSZ without altering its translucency. This is possible because the glass infiltrates the grain boundaries via capillary pressure, resulting in a glass-rich surface layer. This layer with a gradient of elastic properties is capable of reducing the surface stress and transferring it into the interior, which increases the flexural strength. The glass is also able to reduce the population of defects producing a more homogeneous surface [17].

Literature reported the TP of human enamel and dentin (1 mm thickness) to be 18.7 and 16.4 , respectively [25]. Table I shows that a large range of TP values was found in the studies for each generation of zirconia, mainly due to methodological differences. Therefore, it is not possible to compare the results of different studies. Nevertheless, most investigations agree that the translucency of the $3^{\text {rd }}$ and $4^{\text {th }}$ generations of zirconia is superior to the $1^{\text {st }}$ and $2^{\text {nd }}$ generations. Yet, all zirconia-based ceramics are still considered semi-translucent materials, which may limit its indication for restorations with high aesthetic demand [19, 20].

\section{CONCLUSIONS}

$3^{\text {rd }}$ and $4^{\text {th }}$ generations of zirconia-based ceramics show improvements in optical properties when compared to $1^{\text {st }}$ and $2^{\text {nd }}$ generations. The translucency parameter (TP) values were lower and less dependent on thickness for 4Y-PSZ and 5Y-PSZ. In addition, zirconia-based ceramics with high cubic phase content for monolithic restorations have greater translucency than veneered $1^{\text {st }}$ generation zirconia. The addition of lanthanum oxide and the infiltration of feldspathic glass in the zirconia surface proved to be good options to obtain a good balance of optical and mechanical properties, but further studies are needed to investigate its effectiveness.

\section{ACKNOWLEDGEMENT}

The authors thank CNPq for the undergraduate student scholarship.

\section{REFERENCES}

[1] J.R. Kelly, P. Benetti, Aust. Dent. J. 56 (2011) 84.

[2] L.H.D. Silva, E. Lima, R.B.P. Miranda, S.S. Favero, U. Lohbauer, P.F. Cesar, Braz. Oral. Res. 31 (2017) e58.

[3] Y. Zhang, B.R. Lawn, J. Dent. Res. 97 (2018) 140.

[4] Y.D. Belo, Q.N. Sonza, M. Borba, A.D. Bona, Cerâmica 59, 352 (2013) 633.

[5] Q.N. Sonza, A. Della Bona, O.E. Pecho, M. Borba, J. Esthet. Restor. Dent., in press.

[6] M. Borba, M.D. de Araujo, K.A. Fukushima, H.N. Yoshimura, P.F. Cesar, J.A. Griggs, A. Della Bona, Dent. Mater. 27 (2011) 710.

[7] R. Ottoni, M. Borba, Cerâmica 64, 372 (2018) 547.

[8] J. Chevalier, Biomaterials 27 (2006) 535.

[9] I. Denry, J.R. Kelly, Dent. Mater. 24 (2008) 299.

[10] B.E. Pjetursson, I. Sailer, N.A. Makarov, M. Zwahlen, D.S. Thoma, Dent. Mater. 31 (2015) 624.

[11] I. Sailer, N.A. Makarov, D.S. Thoma, M. Zwahlen, B.E. Pjetursson, Dent. Mater. 31 (2015) 603.

[12] B. Stawarczyk, K. Frevert, A. Ender, M. Roos, B. Sener, T. Wimmer, J. Mech. Behav. Biomed. Mater. 59 (2016) 128. [13] F. Zhang, M. Inokoshi, M. Batuk, J. Hadermann, I. Naert, B. Van Meerbeek, J. Vleugels, Dent. Mater. 32 (2016) e327.

[14] M. Borba, T.K. Okamoto, M. Zou, M.R. Kaizer, Y. Zhang, Dent. Mater. 37 (2020) 158.

[15] S. Vardhaman, M. Borba, M.R. Kaizer, D. Kim, Y. Zhang, Dent. Mater. 36 (2020) 1407.

[16] N. Kolakarnprasert, M.R. Kaizer, D.K. Kim, Y. Zhang, Dent. Mater. 35 (2019) 797. 
[17] L. Mao, M.R. Kaizer, M. Zhao, B. Guo, Y.F. Song, Y. Zhang, J. Dent. Res. 97 (2018) 1222.

[18] N. Sen, S. Isler, J. Prosthet. Dent. 123 (2020) 761.

[19] A. Vichi, M. Carrabba, R. Paravina, M. Ferrari, J. Esthet. Restor. Dent. 26 (2014) 224.

[20] T.A. Sulaiman, A.A. Abdulmajeed, T.E. Donovan, A.V. Ritter, P.K. Vallittu, T.O. Narhi, L.V. Lassila, Dent. Mater. 31 (2015) 1180.

[21] S. Turgut, J. Esthet. Restor. Dent. 32 (2020) 26.
[22] S.E. Elsaka, J. Prosthodont. 28 (2019) e279.

[23] E. Camposilvan, R. Leone, L. Gremillard, R. Sorrentino,

F. Zarone, M. Ferrari, J. Chevalier, Dent. Mater. 34 (2018) 879.

[24] J. Yan, M.R. Kaizer, Y.Zhang, J. Mech. Behav. Biomed. Mater. 88 (2018) 170.

[25] B. Yu, J.S. Ahn, Y.K. Lee, Acta Odontol. Scand. 67 (2009) 64.

(Rec. 23/12/2020, Rev. 23/03/2021, Ac. 05/04/2021) 